\title{
Solid-State Photochemical Reaction of Multisubstituted Thymine Derivatives
}

\author{
Akihiro Udagawa ${ }^{\dagger}$ Priscilla Johnston, ${ }^{\S}$ Hidehiro Uekusa,,$"$ Hideko Koshima,,
}

\section{Kei Saito ${ }^{*}$ and Toru Asahi ${ }^{*}, t, \|$}

†Department of Advanced Science and Engineering, Graduate School of Advanced Science and Engineering, Waseda University, 3-4-1 Okubo, Shinjuku-ku, Tokyo 169-8555, Japan.

${ }^{\S}$ School of Chemistry, Monash University, Clayton, VIC 3800, Australia.

*Department of Chemistry and Materials Science, Tokyo Institute of Technology, Ookayama 2-12-1, Meguro-ku, Tokyo 152-8551, Japan.

"Research Organization for Nano \& Life Innovation, Waseda University, 513 Wasedatsurumakicho, Shinjuku-ku, Tokyo 162-0041, Japan.

*Corresponding author: email: kei.saito@ monash.edu / tasahi@waseda.jp

Totals -6 pages, 6 figures 


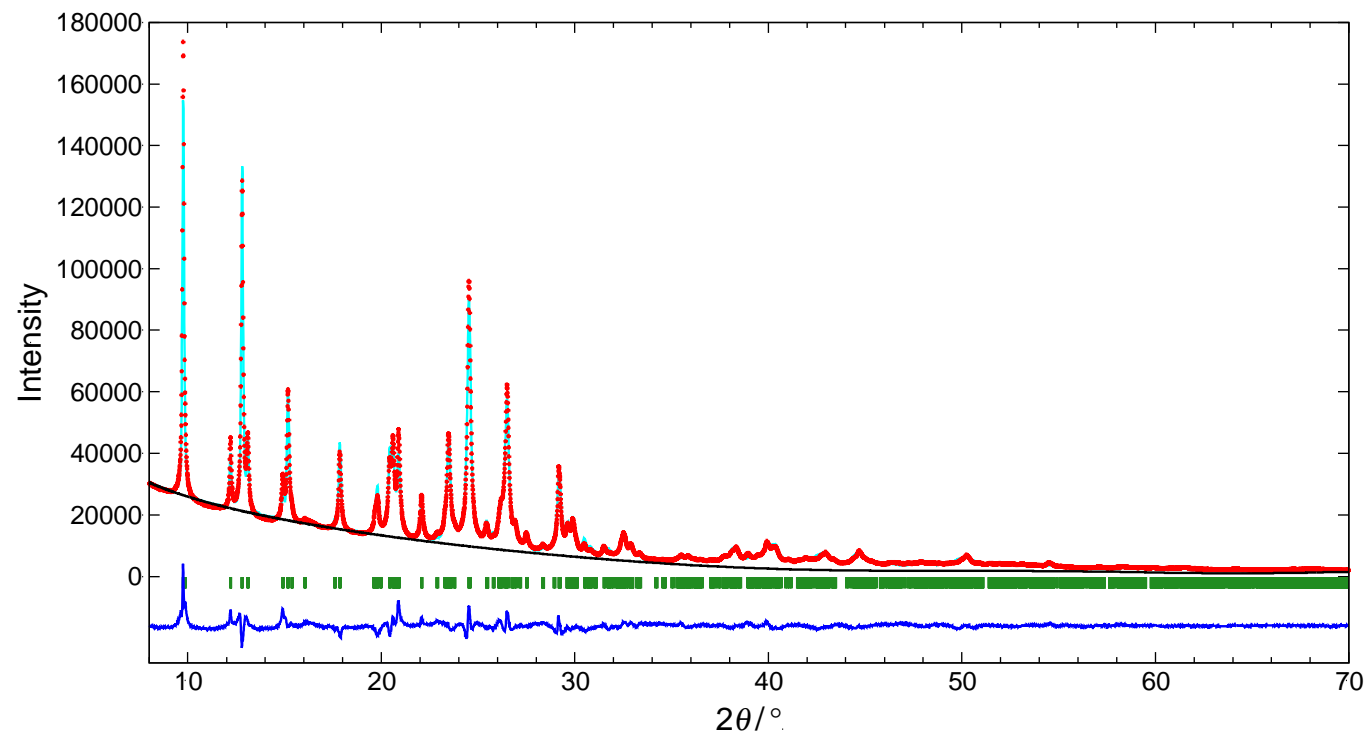

Figure S1. Results from powder X-ray diffraction analysis of $\mathbf{2 T p}$ by Rietveld refinement. Each plot shows the experimental powder X-ray diffraction profile (red dots), the calculated powder X-ray diffraction profile (light-blue solid line), the background profile (black, solid line) and the difference profile (blue, lower line). Green tick marks indicate peak positions. 


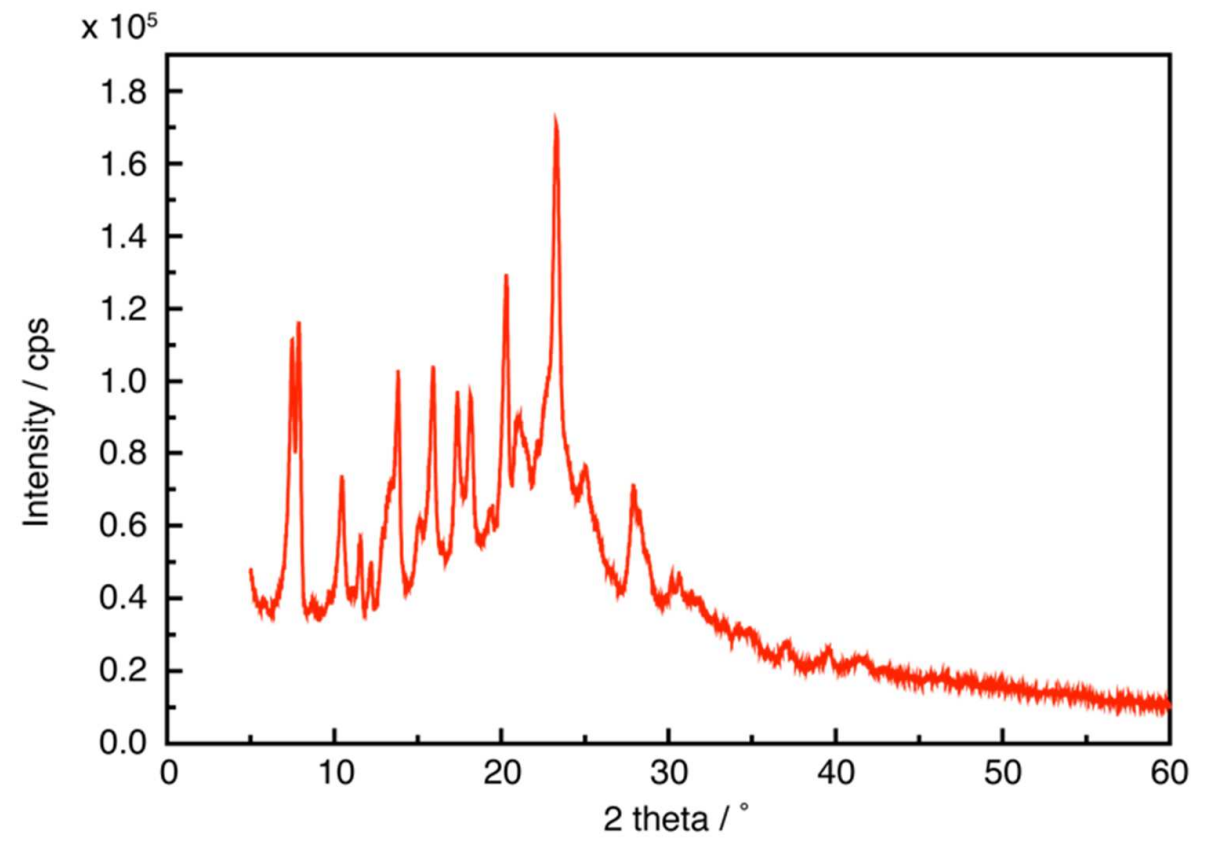

Figure S2. XRD pattern of 2To obtained by slow evaporation of ethanol solution.

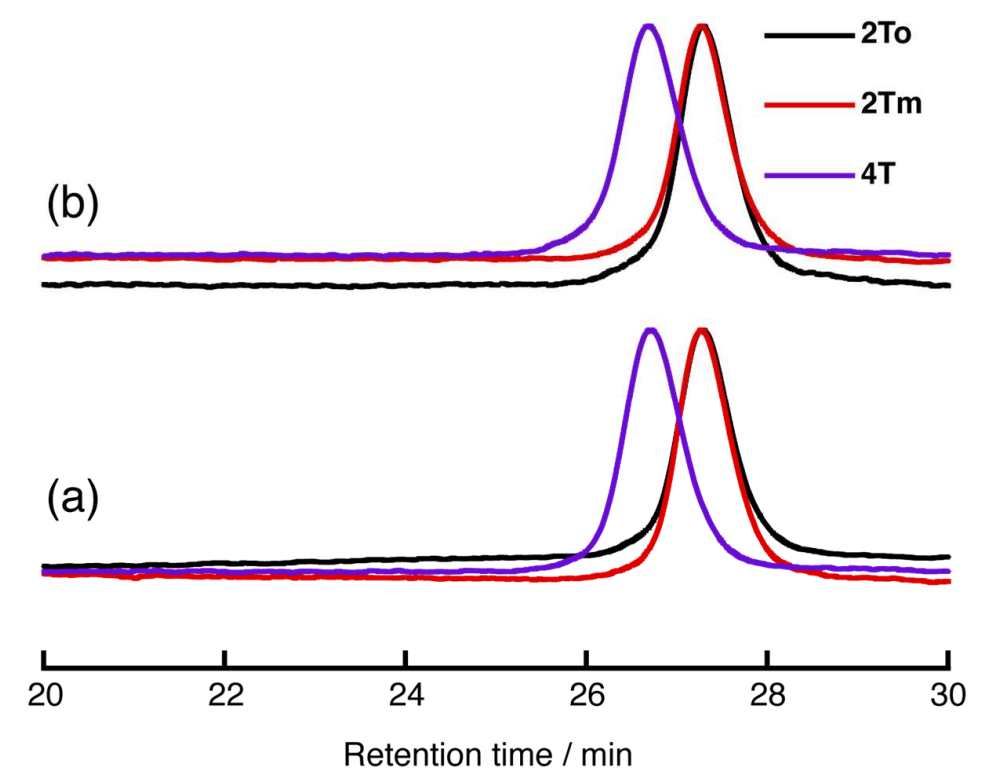

Figure S3. GPC chromatogram of 2To, 2Tm and 4T; (a) before and (b) $48 \mathrm{~h}$ after UV irradiation. 
(a)

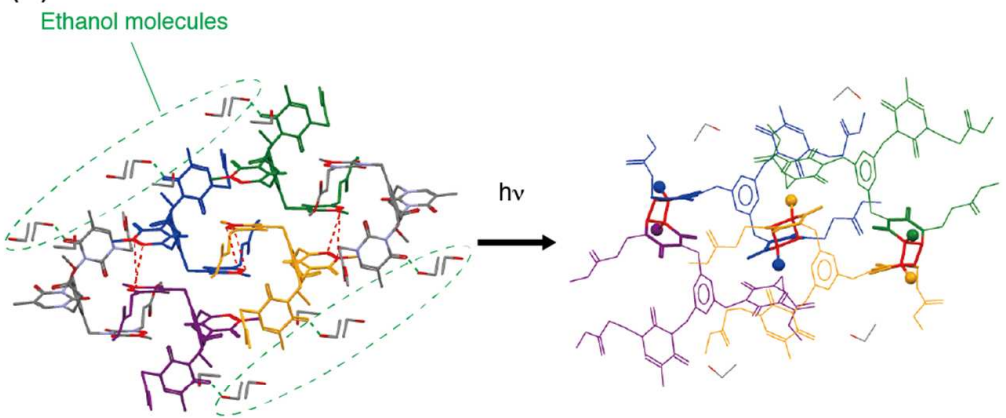

(b)

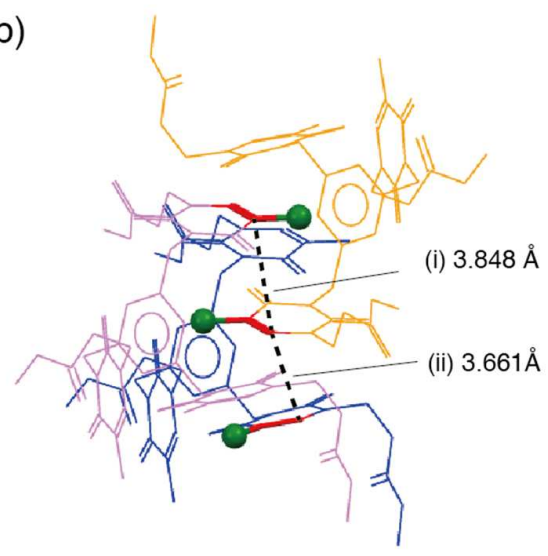

(i)

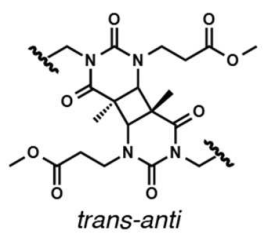

(ii)

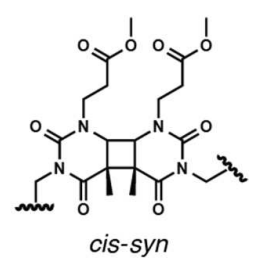

(c)
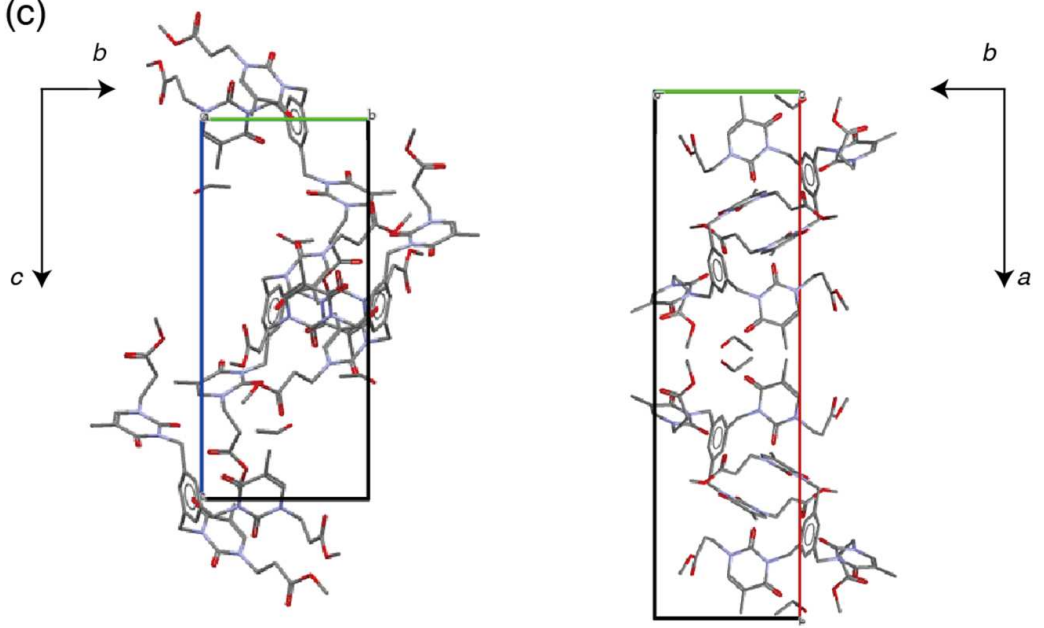

Figure S4. (a) Enlarged Figure 5 (c) in the main text; (b) anticipated stereochemistry of the photoproduct of 3T (three molecules of 3T were colored with pink, yellow and blue, reactive olefins were colored red, and a carbon atom of methyl group in each molecules were illustrated by green ball for clarity); (c) crystal packing of 3T viewed along the $a$ axis (left) and the $c$ axis (right), molecules were reduced to half to be shown for clarity. 


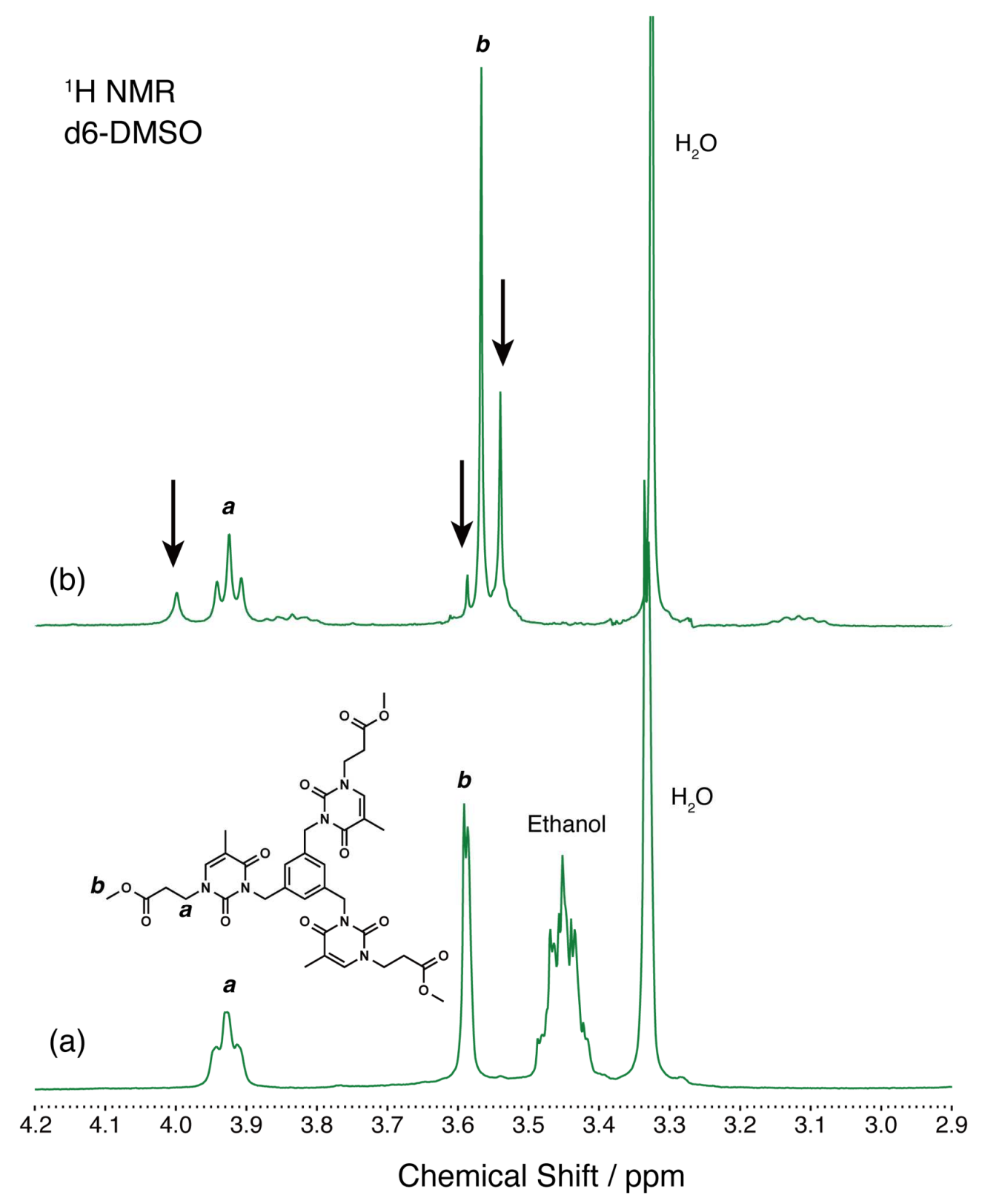

Figure S5. ${ }^{1} \mathrm{H}$ NMR chart of $\mathbf{3 T}$ crystals (a) before and (b) $48 \mathrm{~h}$ after UV irradiation. 


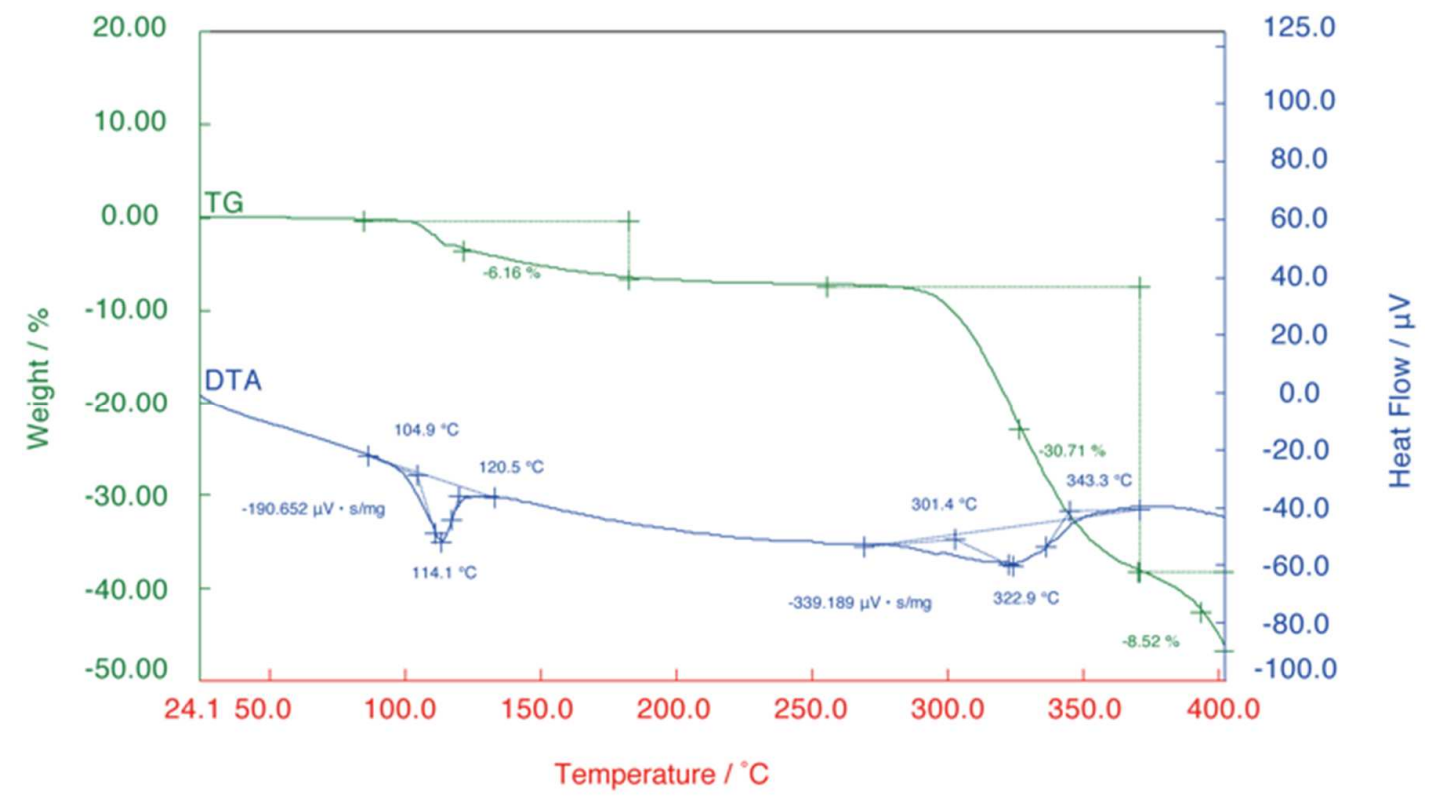

Figure S6. TG-DTA chart of the crystalline 3T with included ethanol molecule. 